\title{
Estimation of Extreme Roll Motion Using the First Order Reliability Method
}

\author{
Choi, Ju-hyuck; Jensen, Jørgen Juncher; Nielsen, Ulrik Dam
}

Published in:

Practical Design of Ships and Other Floating Structures. PRADS 2019.

Link to article, DOI:

/10.1007/978-981-15-4624-2_41

Publication date:

2021

Document Version

Peer reviewed version

Link back to DTU Orbit

Citation (APA):

Choi, J., Jensen, J. J., \& Nielsen, U. D. (2021). Estimation of Extreme Roll Motion Using the First Order Reliability Method. In T. Okada, Y. Kawamura, \& K. Suzuki (Eds.), Practical Design of Ships and Other Floating Structures. PRADS 2019. (pp. 682-690). Springer. Lecture Notes in Civil Engineering Vol. 63 https://doi.org//10.1007/978-981-15-4624-2_41

\section{General rights}

Copyright and moral rights for the publications made accessible in the public portal are retained by the authors and/or other copyright owners and it is a condition of accessing publications that users recognise and abide by the legal requirements associated with these rights.

- Users may download and print one copy of any publication from the public portal for the purpose of private study or research.

- You may not further distribute the material or use it for any profit-making activity or commercial gain

- You may freely distribute the URL identifying the publication in the public portal 


\title{
Estimation of Extreme Roll Motion using the First Order Reliability Method
}

\author{
Ju-hyuck Choi ${ }^{1}$, Jørgen Juncher Jensen ${ }^{2}$, Ulrik Dam Nielsen ${ }^{2,3}$ \\ ${ }^{1}$ Hyundai Heavy Industries, Co., Ltd., Korea \\ ${ }^{2}$ Dept. of Mechanical Engineering, Technical University of Denmark, Kgs. Lyngby, Denmark \\ ${ }^{3}$ Centre for Autonomous Marine Operations (NTNU AMOS), Norwegian University of Science \\ and Technology (NTNU), NO-7491 Trondheim, Norway \\ choijh@hhi.co.kr
}

\begin{abstract}
Extreme value statistics can often be based on the assumption that exceedance events of a high threshold level are statistically independent and identically distributed (i.i.d. process), which further implies the Poisson assumption to be valid. This makes it possible to express the extreme response statistics through the mean up-crossing rate. For non-linear processes, analytic expressions of the mean up-crossing rate do not in general exist. Reliable statistics of mean up-crossing rate based on the brute-force approach, e.g. Monte Carlo simulation (MCS) require long time domain simulations considering a number of different ensemble input. The associated computations can be very time consuming especially when a detailed physical (e.g. hydrodynamic) model is applied. The First Order Reliability Method (FORM) has previously been found efficient for estimation of extreme value prediction of stationary stochastic time domain processes, However, if the non-linearity in a response is significant, the accuracy of the FORM linearized mean up-crossing rate can be limited.

The present work attempts to improve the extreme value prediction for nonlinear parametric roll motions of ships based on applications of the FORM approach and suggests a model for the mean up-crossing rate for strong non-linear response, validated by comparing with MCS results.
\end{abstract}

Keywords: Extreme value statistics, Poisson up-crossing, intact stability of ships

\section{Introduction}

The Poisson assumption is often used for extreme value prediction for stationary stochastic processes and requires only two parameters: the up-crossing rate and the time duration of the response. For a linear system, the up-crossing rate is a product of the mean zero up-crossing rate and an exponential term depending on the threshold level in terms of the reliability index. For non-linear systems, the determination of the up-crossing rate is more complicated, and moreover, the reliability index is not a linear function of the threshold level. The question is then to determine the reliability 
index and to establish an accurate model of the up-crossing rate for non-linear systems. The First Order Reliability Method (FORM) can be used to determine the reliability index and it has been shown in several papers that often a good agreement is found as compared to direct Monte Carlo Simulations (MCS), e.g. Der Kiureghian (2000), Jensen (2015), Choi et al. (2017) and Jensen et al. (2017). The reason is that the reliability index is calculated using the exact non-linear limit state function. The mean zero up-crossing rate can be estimated from the linearized FORM solution, Jensen and Capul (2006), Fujimura and Der Kiureghian (2007), Garrè and Der Kiureghian (2010). However, due to the linearization it might not be as accurate as the reliability index, especially for systems with very strong (or bifurcation type of) nonlinearity.

This paper attempts to improve the estimation of the up-crossing rate for non-linear response based on the framework of the First Order Reliability Method. As a case study, large parametric roll motions of a ship are considered in long-crested head sea seas, which is mainly a non-linear bifurcation problem without any linear components. Two models for the up-crossing rate are examined, both making use of the reliability index from FORM. The results are in line with findings based on Monte Carlo Simulations.

\section{First Order Reliability method (FORM)}

Extreme value statistics can often be based on the assumption of independent peaks implying a Poisson model. For stationary processes the probability that the response $X(t)$ exceeds the level $x_{0}$ during the time $T$ then becomes

$$
P\left[\max _{T} X(t)>x_{0}\right]=1-\exp \left(-v^{+}\left(x_{0}\right) T\right)
$$

For linear (Gaussian) systems, the mean up-crossing rate $v^{+}\left(x_{0}\right)$ takes the form

$$
v^{+}\left(x_{0}\right)=\frac{1}{2 \pi} \sqrt{\frac{m_{2}}{m_{0}}} \exp \left(-\frac{\left(x_{0}-x_{\text {mean }}\right)^{2}}{2 m_{0}}\right)
$$

Eq. (2) requires calculation of the spectral moments $m_{0}, m_{2}$ and therefore also a spectral formulation of the response. For non-linear systems, a linearization around the design point $x_{0}$ using FORM can often provide a useful estimate of the mean upcrossing rate, e.g. Jensen and Capul (2006), Fujimura and Der Kiureghian (2007), Garrè and Der Kiureghian (2010). FORM searches the solution $\underline{u}^{*}$ of the limit state problem: 


$$
\begin{aligned}
& G(\underline{u}) \equiv x_{0}-X(0 \mid \underline{u})=0 \\
& \underline{u}^{*}: \text { Minimize } \underline{u}^{T} \underline{u} ; \text { Subject to } G(\underline{u})=0
\end{aligned}
$$

where $\underline{u}^{T}=\left[u_{1}, u_{2},, \ldots, u_{n}\right]$ are uncorrelated standard normal distributed variables defining the stochastic variations of the input $Y(t \mid \underline{u})$, e.g. the wave elevation and gust wind speed. The time instance $\mathrm{t}=0$, must be so far away from the initial conditions that these do not influence the response. For parametric roll 300s was found in Jensen (2007) to be sufficient.

As shown in Jensen (2011) the spectral density $S_{x}(\omega)$ of the FORM linearized response becomes

$$
S_{x}\left(\omega_{i}\right) d \omega=\left(\left.\frac{\partial X}{\partial u_{i}}\right|_{\underline{\underline{u}}=\underline{u}^{*}}\right)^{2}=e_{i}^{2}
$$

when each $u_{i}$ is associated with a sinusoidal linearized response variation in time with frequency $\omega_{i}$. Hence,

$$
\begin{aligned}
& x_{\text {mean }}=x_{0}-\underline{e}^{T} \underline{u}^{*} \\
& m_{0}=\int S_{x}(\omega) d \omega=\sum_{i=1}^{n} e_{i}^{2}=\|e\|^{2} \\
& m_{2}=\int \omega^{2} S_{x}(\omega) d \omega=\sum_{i=1}^{n} \omega_{i}^{2} e_{i}^{2}
\end{aligned}
$$

It is noted that both $m_{0}$ and $m_{2}$ depend on the design point $\underline{u}^{*}$ and thereby on $x_{0}$. Eq. (2) can then be written, using Eq. (5)

$$
v^{+}\left(x_{0}\right)=\frac{1}{2 \pi \beta} \sqrt{\sum_{i=1}^{n} \omega_{i}^{2} u_{i}^{* 2}} \exp \left(-\frac{\beta^{2}}{2}\right)
$$

where the reliability index $\beta$ is defined as

$$
\beta\left(x_{0}\right)=\sqrt{\underline{u}^{* T} \underline{u}^{*}}=\frac{\underline{e}^{T} \underline{u}^{*}}{\|e\|}
$$


The autocorrelation function of the linearized FORM solution becomes

$$
R\left(t \mid \underline{u}^{*}\right)=\int S_{x}(\omega) \cos \omega t d \omega=\sum_{i=1}^{n} e_{i}^{2} \cos \omega_{i} t
$$

The corresponding most probable linear response is then

$$
X\left(t \mid \underline{u}^{*}\right)_{\text {Linear }}=\frac{x_{0}}{m_{0}} R\left(t \mid \underline{u}^{*}\right)
$$

A comparison between $X\left(t \mid \underline{u}^{*}\right)_{\text {Linear }}$ and $X\left(t \mid \underline{u}^{*}\right)$ provides an indication of how close the FORM linearization is to the real limit state surface $G(\underline{u})$ around the design point $\underline{u}^{*}$.

Choi and Jensen (2019) have investigated the accuracy of Eq. (6) for two types of ship roll motions: dead ship conditions in beam sea and parametric rolling in longcrested head sea. For the dead ship case, comparison between Eq. (9) and $X\left(t \mid \underline{u}^{*}\right)$ shows good agreement, whereas the opposite was the case for parametric rolling, where a significant difference was found between the dominating period in the two results for the most probable response. Hence, Eq. (6) as based on the FORM linearization might not be accurate for highly non-linear responses and alternative expressions are proposed in the next section.

Parametric roll responses are obtained by solving 6 DOF equation of motion for a Post-Panamax container ship. Radiation forces are calculated by using the impulse response function approach, and the nonlinearities are considered in the damping, the Froude-Krylov and restoring forces. The waves are generated using JONSWAP spectrum with the significant wave height of $3.25 \mathrm{~m}$, and the zero up-crossing period of $13.1 \mathrm{sec}$. Further details can be found in Choi and Jensen (2019). The probability Density Function (PDF) of the calculated roll response are shown in Fig.1. A strong non-linearity can be seen from the shape of PDFs as they do not follow the Gaussian distribution, and moreover different wave realizations produce different distributions.
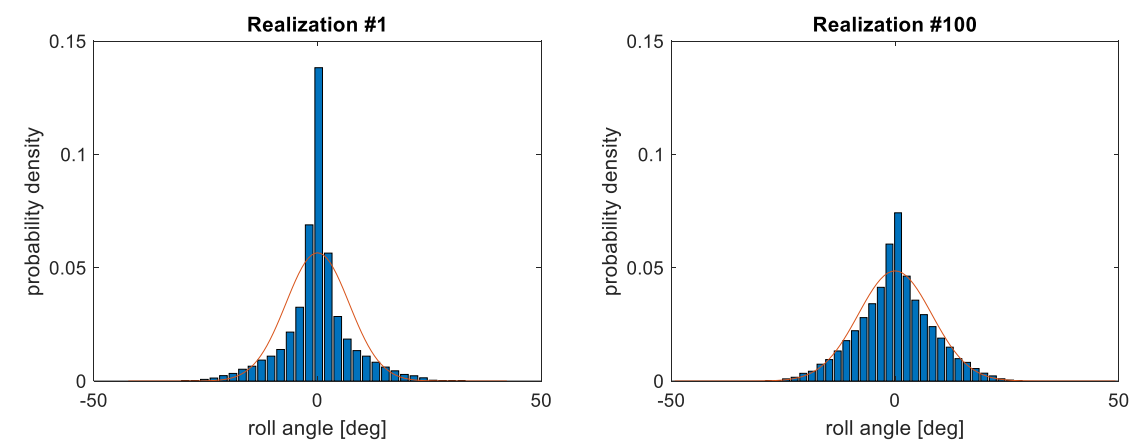

Fig.1. Probability density function of parametric roll angle. Red curve: Gaussian distribution 


\section{Up-crossing rate}

For a stochastic process the up-crossing rate $v^{+}(x)$ can in general be calculated as

$$
v^{+}(x)=\int_{\dot{x}=0}^{\infty} p(x, \dot{x}) \dot{x} d \dot{x}
$$

For stationary processes, the covariance of $x$ and $\dot{x}$ is zero. For some processes, this implies that $x$ and $\dot{x}$ are (nearly) uncorrelated. In that case, the joint and marginal density functions of $x$ and $\dot{x}$ are related by $p(x, \dot{x})=p_{x}(x) p_{\dot{x}}(\dot{x})$ and the up-crossing rate becomes

$$
v^{+}(x)=v^{+}(0) \frac{p_{x}(x)}{p_{x}(0)}
$$

The reliability index $\beta$ is defined through the standard normal distribution function $\Phi$

$$
\int_{x}^{\infty} p_{x}(u) d u \equiv \Phi(-\beta)=\int_{\beta(x)}^{\infty} \frac{1}{\sqrt{2 \pi}} \exp \left(-\frac{1}{2} u^{2}\right) d u
$$

Thus, by differentiation

$$
p_{x}(x)=\frac{1}{\sqrt{2 \pi}} \exp \left(-\frac{1}{2} \beta(x)^{2}\right) \frac{d \beta}{d x}
$$

Hence, with $\beta^{\prime} \equiv d \beta / d x$

$$
v^{+}(x)=v^{+}(0) \exp \left(-\frac{1}{2}\left(\beta(x)^{2}-\beta(0)^{2}\right) \frac{\beta^{\prime}(x)}{\beta^{\prime}(0)}\right.
$$

For symmetric processes, e.g. parametric roll, $\beta(0)=0$ yielding

$$
v^{+}(x)=v^{+}(0) \frac{\beta^{\prime}(x)}{\beta^{\prime}(0)} \exp \left(-\frac{1}{2} \beta(x)^{2}\right)
$$

Eq. (13) depends only on the assumption of statistical independence between $x, \dot{x}$. For Gaussian processes $\beta$ varies linearly with $x$ and the usual formula 


$$
v^{+}(x)=v^{+}(0) \exp \left(-\frac{1}{2} \beta(x)^{2}\right)
$$

is obtained. For non-linear processes the effective up-crossing rate of the mean level in Eq. (13)

$$
v_{\text {mean }}^{+}(x)=v^{+}(0) \frac{\beta^{\prime}(x)}{\beta^{\prime}(0)}
$$

depends solely on $x$ in contrast to the true mean up-crossing rate, i.e. Eq. (10).

Without the assumptions of statistical independence, Eq. (14) is replaced by

$$
v_{\text {mean }}^{+}(x)=v^{+}(0) \frac{\beta^{\prime}(x) f(x)}{\beta^{\prime}(0) f(0)}
$$

where

$$
f(x)=\int_{0}^{\infty} p_{\dot{x}}(\dot{x} \mid x) \dot{x} d \dot{x}
$$

It could be noted that the FORM result, Eq. (6), for $v^{+}(x)$ does not assume statistical independence of $x, \dot{x}$.

Based on Eqs (11)-(16) two semi-empirical formulas for the up-crossing rate are considered. The first is

$$
\text { Model-A: } v^{+}(x)=v_{0}^{+} \exp \left(-\frac{1}{2} \beta(x)^{2}\right)
$$

The differences between in Eq.(17) and Eq. (2) are in the introduction of $v_{0}$ and $\beta$. The up-crossing rate relative to the mean level $v_{0}$ can be obtained either from a small number of simulations or estimated from the roll natural frequency $\omega_{\text {roll }}$, i.e. $v_{0}=$ $\omega_{\text {roll }} / 2 \pi$. In Eq. (17), $\beta$ is used due to the mapping of the non-linear process to the standard normal distribution, Eq.(12). From Monte Carlo Simulations the reliability index can be calculated as

$$
\beta_{M C S}\left(x_{i}\right)=-\Phi^{-1}\left(1-\frac{i-1}{M}\right) ; \quad 1=1,2, \ldots, M
$$

where $x_{i}$ are the ordered ensemble samples of roll response taken at a fixed time instance: $x_{i} \leq x_{i+1} ; i=1,2, \ldots, M$., (e.g. Jensen et al. 2017). 
The second model is based on Eq. (15), and for better estimation of extreme values, a reference point $x_{\text {ref }}$ introduced

Model-B: $v^{+}\left(x_{0}\right)=v^{+}\left(x_{r e f}\right) \frac{\beta^{\prime}\left(x_{0}\right) f(x)}{\beta^{\prime}\left(x_{r e f}\right) f\left(x_{r e f}\right)} \exp \left(-\frac{1}{2}\left(\beta\left(x_{0}\right)^{2}-\beta\left(x_{r e f}\right)^{2}\right)\right)(19)$

where, $x_{\text {ref }}$ is selected as large as possible within the range where the MCS upcrossing rate, $v^{+}\left(x_{r e f}\right)$ is accurate. The derivative $\beta^{\prime}(x)$ of the reliability index is calculated numerically from $\beta(x)$.

An analytic expression for the conditional partial probability density function $p_{\dot{x}}(\dot{x} \mid x)$ does not exist in general for non-linear processes. Therefore, $f\left(x_{0}\right)$ in Eq. (16) is calculated from the time histories by taking average of the velocities $\dot{x}$ at given threshold levels. If $\dot{x}$ and $x$ are independent, then $f\left(x_{0}\right) / f\left(x_{r e f}\right)=1$.

The remaining problem is how to calculate an accurate reliability index $\beta$ for extreme roll angles outside the range that can be covered by MCS. Here, FORM provides $\beta$ values for extreme roll angles efficiently. For the present parametric roll example, the results in Fig. 2, taken from Choi and Jensen (2019), will be used in the validation process of Model-A and Model-B. The figure shows that $\beta_{F O R M}$ is slightly smaller than $\beta_{M C S}$ when large roll angles are considered, implying that the estimated results then will be slightly conservative using $\beta_{F O R M}$ instead of $\beta_{M C S}$. The reliability index $\beta_{\text {FORM }}\left(x_{0}\right)$ for $x_{0} \geq x_{\text {ref }}=28 \mathrm{deg}$ will thus be used in the following.

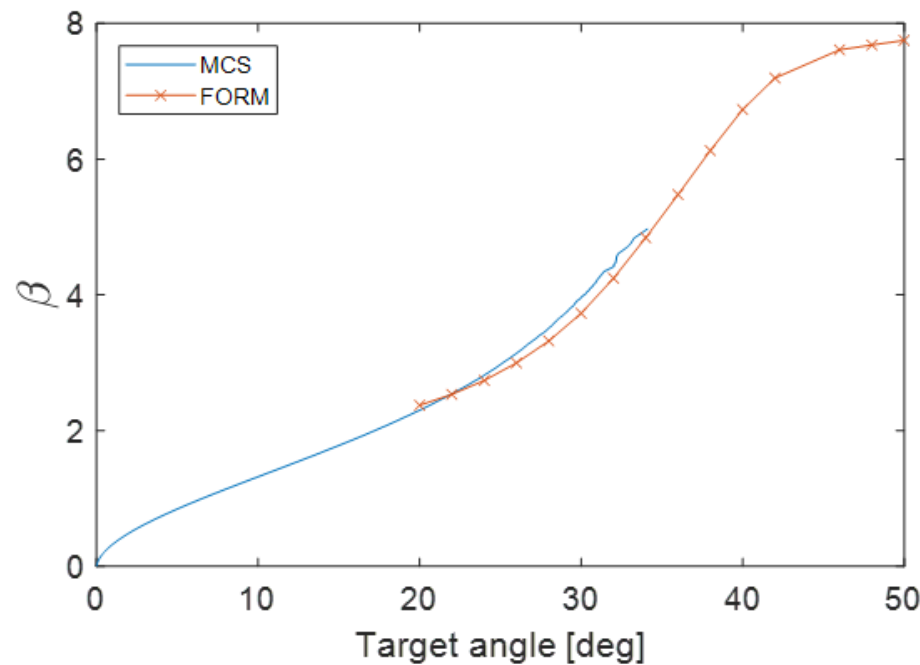

Fig. 2. Reliability index $\beta$ from FORM and MCS, Choi and Jensen (2019) 


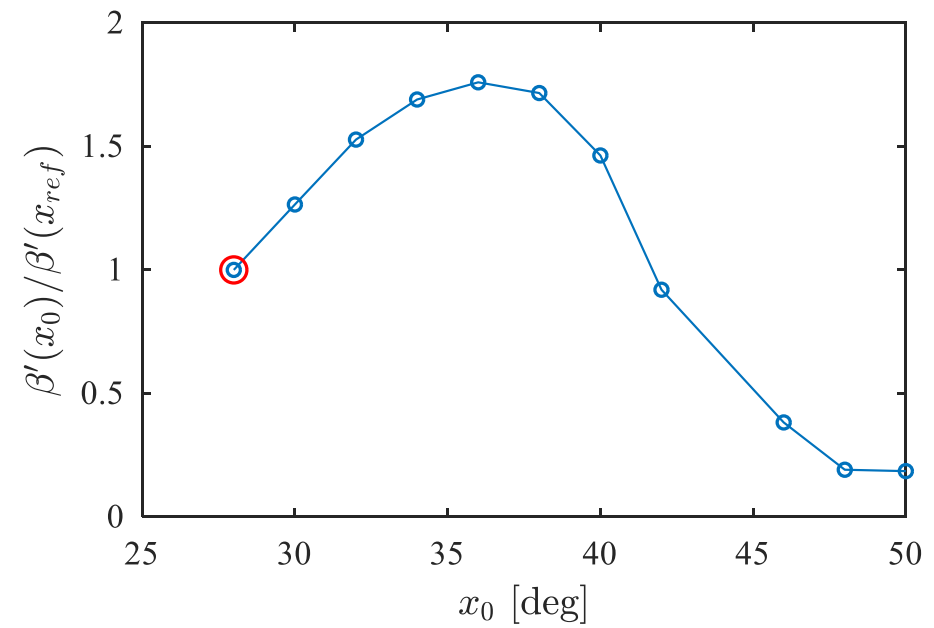

Fig. 3. $\beta^{\prime}\left(\mathrm{x}_{0}\right) / \beta^{\prime}\left(\mathrm{x}_{\mathrm{ref}}\right)$ from $\beta_{\mathrm{FORM}}$ for parametric rolling $\left(\mathrm{x}_{\mathrm{ref}}=28 \mathrm{deg}\right.$.)

The corresponding $\beta^{\prime}\left(x_{0}\right) / \beta^{\prime}\left(x_{\text {ref }}\right)$ curve is shown in Fig. 3. For use in Model-B, $f\left(x_{0}\right)$, Eq.(16), is extrapolated for $x_{0} \geq x_{\text {ref }}=28 \mathrm{deg}$ from the Monte Carlo simulation and shown in Fig. 4. The dotted lines denote $95 \%$ of confidence interval of the $\beta^{\prime}\left(x_{0}\right) / \beta^{\prime}\left(x_{\text {ref }}\right)$. The extrapolation chosen is linear fit based on the mean values in the tail regions, which inevitably leads to a large uncertainty. However, $f\left(x_{0}\right)$ is not that important for the up-crossing rate, which is dominated by exponential term.

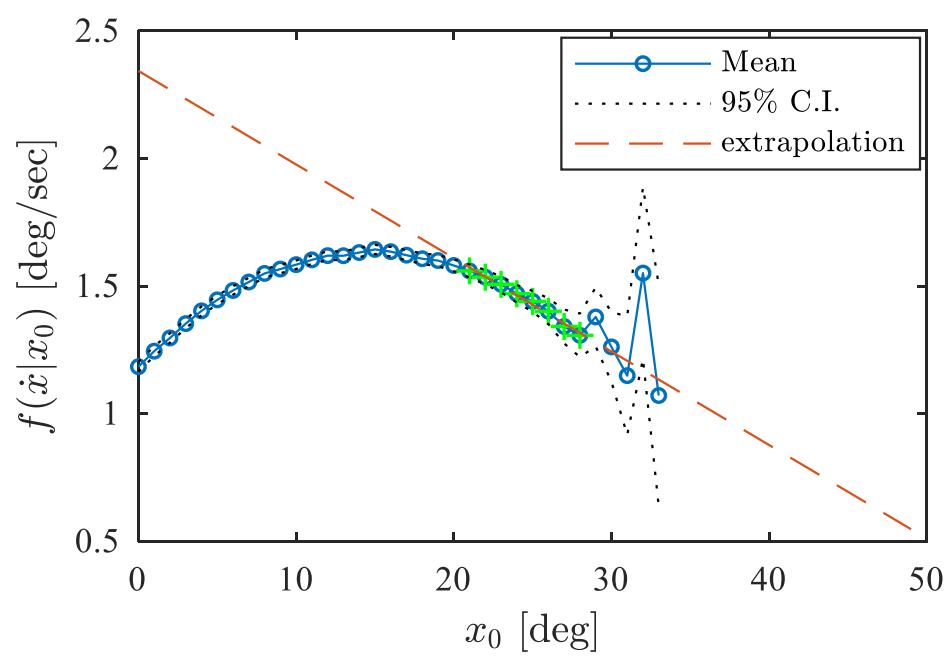

Fig. 4. The extrapolated $f\left(x_{0}\right)$ for parametric rolling obtained from MCS 
Fig. 5 shows the calculated up-crossing rates from the two different models. In general, no significant differences are found. One attractive feature seen from the figure is that both methods $\mathrm{A}$ and $\mathrm{B}$ can capture the complex probability behavior at extreme levels $\left(x_{0}>40 \mathrm{deg}\right.$.) where a strong non-linearity (capsize) causes different response characteristics. This is difficult to obtain by an extrapolation technique and is one of the advantages of the FORM application for extreme value predictions. For larger extreme angles i.e. $x_{0}>40 \mathrm{deg}$, the discrepancies between Model-A and Model$\mathrm{B}$ seem not to be important since the probability levels are very small. It is interesting that Model-A can be used for extreme value predictions with good accuracy as it can be calculated purely from FORM to obtain $\beta_{F O R M}$ and by replacing $v_{0}$ with $\omega_{\text {roll }} / 2 \pi$.

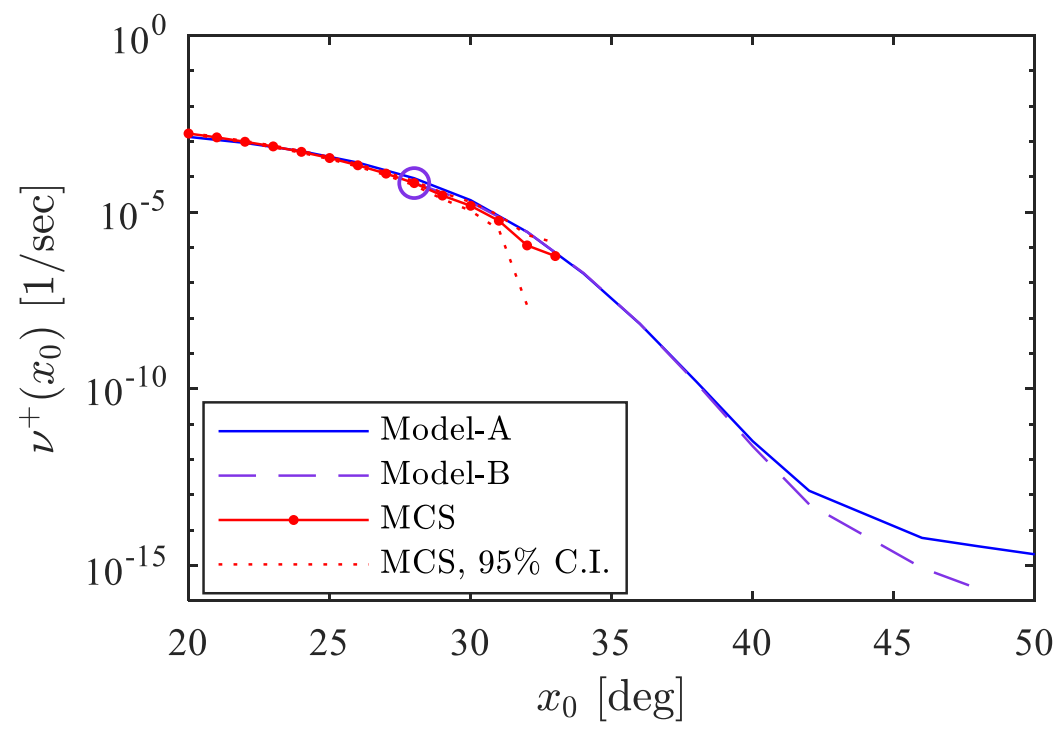

Fig. 5. Estimated up-crossing rates from the present methods

\section{Conclusion}

The purpose of the paper is to investigate the applicability of the Poisson extreme value model for stationary stochastic processes as applied to non-linear roll motion of ships. The probabilistic framework is the First Order Reliability Method (FORM), where previous studies have shown that the reliability index $\beta$ obtained by FORM is very close to the results from direct Monte Carlo Simulations (MCS).

The paper focuses on the up-crossing rate. It was found in Choi and Jensen (2019) that for bifurcation type non-Gaussian processes such as parametric rolling of ships, a significant discrepancy between a Gaussian estimation and Monte Carlo Simulations is observed. Therefore, alternative models for mean up-crossing rate are evaluated in the present study through Monte Carlo Simulations, and it is found that a simple model (Model-A) provides a reasonably good estimation. 


\section{Acknowledgments}

The authors would like to thank Prof. Gabriele Bulian for the fruitful discussions. The work by the third author has been supported by the Research Council of Norway through the Centres of Excellence funding scheme, Project number 223254-AMOS.

\section{References}

Choi, J. hyuck, Jensen, J.J. 2019. Extreme value predictions using FORM for ship roll motions. Mar. Struct. 66, 52-65.

Der Kiureghian, A., 2000. Geometry of random vibrations and solutions by FORM and SORM, Probabilistic Eng Mech. 21,81-90

Fujimura, K., Der Kiureghian, A., 2007. Tail-equivalent linearization method for nonlinear random vibration. Probabilistic Eng. Mech. 22, 63-76.

Garrè, L., Der Kiureghian, A., 2010. Tail-Equivalent Linearization Method in frequency domain and application to marine structures. Mar. Struct. 23, 322-338.

Jensen, J.J., 2007. Efficient estimation of extreme non-linear roll motions using the first-order reliability method (FORM). J. Marine Science and Technology, Vol 12, No. 4, pp 191-202

Jensen, J.J., 2011. Extreme value predictions using Monte Carlo simulations with artificially increased load spectrum. Probabilistic Eng. Mech. 26, 399-404.

Jensen, J.J., 2015. Conditional stochastic processes applied to wave load predictions, Weinblum Memorial Lecture 2014. J. Sh. Res. 59, 1-10. 